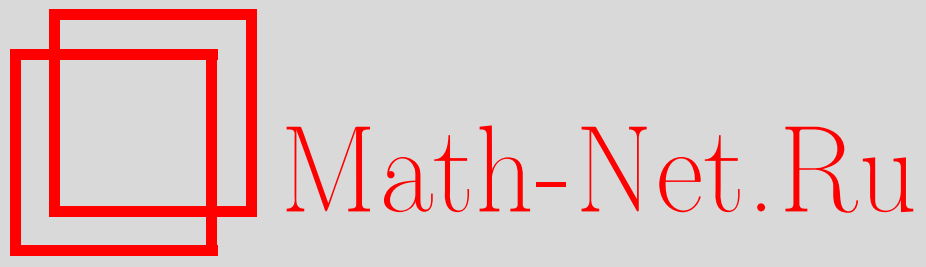

Г. А. Калябин, Наилучшие операторы продолжения для соболевских пространств на полупрямой, Функи. анализ и его прил., 2002, том 36, выпуск $2,28-37$

DOI: https://doi.org/10.4213/faa188

Использование Общероссийского математического портала MathNet.Ru подразумевает, что вы прочитали и согласны с пользовательским соглашением

http://www.mathnet.ru/rus/agreement

Параметры загрузки:

IP: 54.237 .59 .107

26 апреля 2023 г., 04:06:23

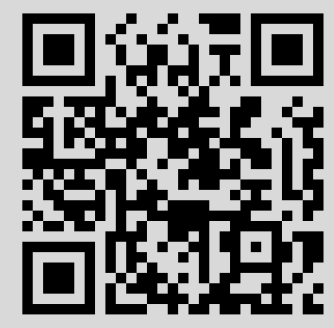


УДК 517.518.237, 512.643.5

\title{
Наилучшие операторы продолжения для соболевских пространств на полупрямой*
}

\author{
(c) 2002. Г. А. КАляБин
}

\section{§1. Обозначения и формулировка основного результата}

Пусть $W_{2}^{m}(I)$ - соболевское пространство всех функций $f(x)$, определенных на интервале $I:=(\alpha, \beta) \subset \mathbb{R}^{1}$, имеющих абсолютно непрерывную производную $f^{(m-1)}(x)$ и таких, что

$$
\|f\|_{W_{2}^{m}(I)}:=\left(\int_{I}\left(|f(x)|^{2}+\left|f^{(m)}(x)\right|^{2}\right) d x\right)^{1 / 2}<\infty .
$$

В [1] построены операторы продолжения $T_{m}: W_{2}^{m}\left(\mathbb{R}_{-}^{1}\right) \rightarrow W_{2}^{m}\left(\mathbb{R}^{1}\right)$, нормы которых не превосходят $8^{m}$. С другой стороны, в [2] было показано, что $W_{2}^{m}\left(\mathbb{R}_{-}^{1}\right)$ содержит такую функцию $f_{m}(x)$, что любая функция $g(x)$, определенная на всей прямой и совпадающая с $f_{m}(x)$ при всех $x<0$, имеет в $W_{2}^{m}\left(\mathbb{R}^{1}\right)$ норму, большую, чем $0,08 m^{-1 / 4} 2^{m}\left\|f_{m}\right\|_{W_{2}^{m}\left(\mathbb{R}_{-}^{1}\right)}$. Нашей целью является устранение имеющегося разрыва между верхними и нижними оценками и установление приведенной ниже асимптотической формулы (точной в логарифмической шкале) для величины

$$
\tau_{m}:=\min \left\|T_{m}\right\|_{W_{2}^{m}\left(\mathbb{R}_{-}^{1}\right) \rightarrow W_{2}^{m}\left(\mathbb{R}^{1}\right)} .
$$

Обозначим через $\mathbf{G}$ так называемую константу Каталана (см., например, [3, 865.03, 48.32]),

$$
\mathbf{G}:=\int_{0}^{\pi / 4} \ln (\operatorname{ctg} x) d x=\sum_{k=0}^{\infty} \frac{(-1)^{k}}{(2 k+1)^{2}}=0,91596559 \ldots .
$$

ТЕОРемА. При $m \rightarrow \infty$ мы имеем $\ln \tau_{m} \approx K_{0} m$, где

$$
K_{0}:=\frac{4}{\pi} \mathbf{G}=1,166243 \ldots=\ln 3,209912 \ldots .
$$

В ходе доказательства будет установлено, что оператор продолжения с наименьшей нормой в рассматриваемом случае является линейным и тесно связан с оператором наилучшей экстраполяции (т.е. продолжения из «одной точки»), задачу исследования которого поставил Л. Д. Кудрявцев. Будет также показано, что $\tau_{m}$ явно выражается через наибольшее и наименьшее собственные значения некоторой матрицы, связанной со специальными матрицами Вандермонда.

Автор выражает глубокую благодарность В. И. Буренкову и Л. Д. Кудрявцеву за постановку задачи и внимание к работе, а также С. И. Похожаеву и другим участникам Семинара по теории функций МИ РАН под руководством С. М. Никольского, Л. Д. Кудрявцева и О. В. Бесова за ряд ценных замечаний. 


\section{§2. Предварительные рассмотрения}

Рассмотрим квадратичный функционал

$$
J_{m}[y]:=\int_{0}^{\infty}\left(\left|y^{(m)}(x)\right|^{2}+|y(x)|^{2}\right) d x=\|y(x)\|_{W_{2}^{m}\left(\mathbb{R}_{+}^{1}\right)}^{2}
$$

и для любого вектора $a:=\left(a_{1}, \ldots, a_{m}\right)$ введем обозначение для минимума квадрата $W_{2}^{m}$-нормы всевозможных экстраполяций с заданными начальными данными:

$$
\psi_{m}(a):=\min \left\{J_{m}[y]: y^{(s-1)}(0)=a_{s}, s \in\{1, \ldots, m\}\right\} .
$$

Экстремалями функционала $J_{m}[y]$ служат решения уравнения Эйлера

$$
(-1)^{m} y^{(2 m)}+y=0,
$$

стремящиеся к 0 при $x \rightarrow+\infty$, которые, следовательно, могут быть представлены как линейные комбинации экспонент (совокупность всех таких линейных комбинаций мы будем обозначать через $\left.Y_{m}\right)$ :

$$
y(x)=\sum_{k=1}^{m} b_{k} e^{\mu_{k} x},
$$

где $\mu_{k}=\mu_{k, m}:=e^{i \pi(2 k+m-1) /(2 m)}, k \in\{1, \ldots, m\}$, суть корни степени $2 m$ из $(-1)^{m+1}$, лежащие в левой полуплоскости. Отметим, что для указанных корней справедливы тождества $\mu_{k}^{m}=i^{m-1}(-1)^{k}, \mu_{k}^{-1}=\bar{\mu}_{k}=\mu_{m+1-k}$, существенно используемые в дальнейшем.

Для произвольной функции $f \in W_{2}^{m}\left(\mathbb{R}_{-}^{1}\right)$ обозначим через $y:=y(f ; x)$ функцию из $W_{2}^{m}\left(\mathbb{R}_{+}^{1}\right)$, имеющую те же самые начальные значения, что и $f$, и дающую минимум функционала $J_{m}[y]$. Тогда продолжение функции $f$ на положительную полуось посредством $y(f ; x)$ и будет (в силу определений) искомым оператором продолжения с наименьшей возможной нормой. Поскольку отображение функции $f \in W_{2}^{m}\left(\mathbb{R}_{-}^{1}\right)$ в множество ее предельных значений $A_{m}:=\left\{\left\{a_{s}\right\}\right\}=$ $\left\{\left\{f^{(s-1)}(0-)\right\}\right\}$ и отображение пространства $A_{m}$ в множество соответствующих экстремалей $Y_{m}$ линейны (так как линейно уравнение Эйлера (7)), описанный оператор наилучшего продолжения также линеен.

Для функций (8) значение $J_{m}[y]$ может быть записано в виде квадратичной формы от коэффициентов $b_{j}$ :

$$
\begin{aligned}
J_{m}[y] & =\int_{0}^{\infty}\left(\left(\sum_{j=1}^{m} b_{j} e^{\mu_{j} x}\right)\left(\sum_{k=1}^{m} \overline{b_{k} e^{\mu_{k} x}}\right)+\left(\sum_{j=1}^{m} b_{j} \mu_{j}^{m} e^{\mu_{j} x}\right)\left(\sum_{k=1}^{m} \overline{\mu_{k}^{m} b_{k} e^{\mu_{k} x}}\right)\right) d x \\
& =\sum_{j=1}^{m} \sum_{k=1}^{m} q_{j, k}^{(m)} b_{j} \bar{b}_{k}, \quad q_{j, k}^{(m)}:=-\frac{1+\mu_{j}^{m} \bar{\mu}_{k}^{m}}{\mu_{j}+\bar{\mu}_{k}}=-\frac{1+(-1)^{j+k}}{\mu_{j}+\bar{\mu}_{k}}, j, k \in\{1, \ldots, m\} .
\end{aligned}
$$

Выражение $J_{m}[y]$ может быть переписано и в терминах начальных значений $y^{(s-1)}(0)=a_{s}$, поскольку коэффициенты $b_{j}$ однозначно определяются из следующей системы линейных алгебраических уравнений:

$$
\sum_{k=1}^{m} \mu_{k}^{j-1} b_{k}=a_{j}, \quad j \in\{1, \ldots, m\},
$$


матрицей которой является классическая матрица Вандермонда

$$
V_{m}=\left\|v_{j, k}^{(m)}\right\|:=\left\|\mu_{k}^{j-1}\right\|
$$

(невырожденная, поскольку все числа $\mu_{k}$ попарно различны), и если обозначить через $\Phi_{m}=\left\|\varphi_{j, k}^{(m)}\right\|, j, k \in\{1, \ldots, m\}$, матрицу, обратную к $V_{m}$ (явные формулы для ее элементов приведены, например в [4]), то в векторной записи мы имеем $V_{m} b=a$ или $b=\Phi_{m} a$. Следовательно,

$$
\psi_{m}(a)=\left(Q_{m} b, b\right)=\left(Q_{m} \Phi_{m} a, \Phi_{m} a\right)=\left(G_{m} a, a\right), \quad G_{m}:=\Phi_{m}^{*} Q_{m} \Phi_{m},
$$

где $Q_{m}$ есть матрица с элементами $q_{j, k}^{(m)}$, определенными в $(9)$, а скобки обозначают обычное скалярное произведение в $m$-мерном унитарном пространстве комплекснозначных векторов.

С другой стороны, для любых двух функций $f(x), g(x)$ из $C^{2 m}[0,+\infty)$, которые стремятся к 0 при $x \rightarrow+\infty$ вместе со своими производными до порядка $2 m-1$, с помощью интегрирования по частям легко проверяется следующее тождество:

$$
\begin{aligned}
& \int_{0}^{\infty} f^{(m)}(x) g^{(m)}(x) d x=-f^{(m)}(0) g^{(m-1)}(0)+f^{(m+1)}(0) g^{(m-2)}(0)-\cdots \\
&+(-1)^{m} f^{(2 m-1)}(0) g(0)+(-1)^{m} \int_{0}^{\infty} f^{(2 m)}(x) g(x) d x .
\end{aligned}
$$

Выбирая здесь в качестве $f(x)$ любую функцию $y(x)$ вида (8), а в качестве $g(x)$ комплексно-сопряженную к ней и учитывая, что в силу уравнения (7) последнее слагаемое, взятое со знаком минус, будет равно интегралу от $|y(x)|^{2}$ по промежутку $[0,+\infty]$, мы приходим к равенству

$$
\begin{aligned}
J_{m}[y] & =\sum_{j=1}^{m}(-1)^{j} y^{(m+j-1)}(0) \overline{y^{(m-j)}(0)} \\
& =i^{m-1} \sum_{j=1}^{m}\left(\sum_{k=1}^{m} b_{k}(-1)^{k} \mu_{k}^{j-1}\right)(-1)^{j} \overline{y^{(m-j)}(0)} .
\end{aligned}
$$

Теперь пусть задан вектор начальных условий $a=\left(a_{1}, \ldots, a_{m}\right)$; тогда вектор коэффициентов $b=\left(b_{1}, \ldots, b_{m}\right)$ получается, как уже отмечалось, умножением $a$ слева на матрицу $\Phi_{m}, b=\Phi_{m} a$, а переход от $b$ к вектору $\tilde{b}:=$ $\left(-b_{1}, b_{2}, \ldots,(-1)^{m} b_{m}\right)$ осуществляется умножением слева на матрицу $P_{m}$, у которой на главной диагонали стоят знакочередующиеся $(-1)^{k}$, а остальные элементы нулевые: $\tilde{b}=P_{m} b$. Переход же от $\tilde{b}$ к вектору с координатами, равными суммам по $k$ в последнем выражении в формуле (14), соответствует умножению на матрицу $V_{m}$. Наконец, вектор с координатами $y^{(m-j)}(0)$ получается умножением $a$ слева на матрицу $S_{m}$ с единицами на побочной диагонали и нулями на всех остальных местах. Таким образом, мы получили другую формулу для квадратичной формы $\psi_{m}(a)$ и ее матрицы $G_{m}($ см. $(5),(6),(14))$ :

$$
\begin{gathered}
\psi_{m}(a)=J_{m}[y]=\left(i^{m-1} V_{m} P_{m} \Phi_{m} a, P_{m} S_{m} a\right)=\left(G_{m} a, a\right), \\
G_{m}=i^{m-1} S_{m} P_{m} V_{m} P_{m} \Phi_{m} .
\end{gathered}
$$




\section{§3. Свойства симметрии матриц $G_{m}$}

Лемма 1. Матрица $G_{m}$ вещественна и симметрична.

ДокАЗАТЕльство. Отметим, во-первых, что матрица $G_{m}$ по определению (см. (12)) эрмитова и положительно определенная. Докажем, что она вещественна. В самом деле, множество тех решений уравнения (7), которые стремятся к 0 при $x \rightarrow+\infty$, в точности совпадает с множеством всех решений дифференциального уравнения $\left(D-\mu_{1}\right) \cdots\left(D-\mu_{m}\right) y(x)=0$ порядка $m$, где $D:=d / d x$. Для четного и нечетного $m$ оно принимает (соответственно) форму

$$
\prod_{k=1}^{m / 2}\left(D^{2}-2 \operatorname{Re} \mu_{k} D+1\right) y=0, \quad(D+1) \prod_{k=1}^{(m-1) / 2}\left(D^{2}-2 \operatorname{Re} \mu_{k} D+1\right) y=0
$$

т. е. $D^{m} y+B_{1} D^{m-1} y+B_{2} D^{m-2} y+\cdots+B_{m-1} D y+y=0$, где все коэффициенты вещественны (даже положительны). Поэтому если все числа $y(0), y^{\prime}(0), \ldots$, $y^{(m-1)}(0)$ вещественны, то функция $y(x)$ будет вещественной при всех $x>0$, а следовательно, $y^{(m)}(0), y^{(m+1)}(0), \ldots, y^{(2 m-1)}(0)$ также вещественны, что в силу первого равенства в $(14)$ означает вещественность всех элементов матрицы $G_{m}$, а отсюда вытекает и ее симметричность.

Доказательство леммы 1 закончено.

Лемма 2. Матрица $G_{m}$ симметрична и относительно побочной диагонали, m.e. $G_{m}=S_{m} G_{m} S_{m}$.

ДокАЗАТЕЛЬСтво. Будем исходить из равенства (см. (15)) $G=i^{m-1} S P V P \Phi$, где для краткости индекс $m$ у рассматриваемых матриц опущен. Введем диагональную матрицу $H:=\operatorname{diag}\left(\mu_{k}^{m-1}\right)$. Напомним, что умножение некоторой квадратной матрицы на матрицу $S$ слева (справа) равносильно ее отражению относительно срединной горизонтали (вертикали), а умножение слева (справа) на диагональную матрицу означает умножение каждой строки (столбиа) на соответствующие диагональные элементы; поэтому для матриц $V S$ и $S V$ имеют место соотношения (см. (11) и определение $\mu_{k}$ после формулы (8))

$$
V S=\left\|\mu_{m+1-k}^{j-1}\right\|=\left\|\mu_{k}^{1-j}\right\|, \quad S V=\left\|\mu_{k}^{m-j}\right\|=V S H \Longleftrightarrow \Phi S=H^{-1} S \Phi .
$$

Далее, имеем цепочку тождеств

$$
\begin{aligned}
S G S & =(-i)^{m-1} S P(S V) P(\Phi S)=(-i)^{m-1} S P(V S H) P\left(H^{-1} S \Phi\right) \\
& =(-i)^{m-1} S P V(S P S) \Phi=i^{m-1} S P V P \Phi=G,
\end{aligned}
$$

где мы учли перестановочность диагональных матриц $H$ и $P$, а также приняли во внимание простые матричные тождества $S P=(-1)^{m-1} P S, S S=P P=$ $E:=\mathrm{id}_{m}$.

Доказательство леммы 2 закончено.

Наиболее неожиданным и весьма существенным является, однако, следующее свойство, в котором содержится, в частности, утверждение о подобии матрицы $G_{m}$ своей обратной.

ЛЕмма 3. $G_{m}^{-1}=P_{m} G_{m} P_{m}$. 
ДокАЗАТЕЛЬСтво. Непосредственно из формулы (15), леммы 2 и тождеств, использовавшихся в ее доказательстве, вытекает требуемая цепочка равенств:

$$
\begin{aligned}
G^{-1} & =(-i)^{m-1}(S P V P \Phi)^{-1}=(-i)^{m-1} V P \Phi P S \\
& =i^{m-1} P S(S P V P \Phi) S P=P(S G S) P=P G P .
\end{aligned}
$$

Следующие утверждения непосредственно вытекают из определений и доказанной леммы 3.

Следствие 1. Спектры матриц $G_{m} u G_{m}^{-1}$ совпадают. В частности, наибольшее и наименьшее собственные значения матрицы $G_{m}$ удовлетворяют равенству $\lambda_{\min }\left(G_{m}\right)=\lambda_{\max }^{-1}\left(G_{m}\right)$, причем если $\xi=\left(\xi_{1}, \ldots, \xi_{m}\right)-$ собственный вектор, отвечающий $\lambda_{\max }\left(G_{m}\right)$, то вектор $P_{m} \xi=\left(-\xi_{1}, \xi_{2}, \ldots,(-1)^{m} \xi_{m}\right)$ также будет собственным и отвечающим $\lambda_{\max }^{-1}\left(G_{m}\right)$.

Следствие 2. Максимум $\Omega_{m}$ и минимум $\omega_{m}$ квадратичной формы $\psi_{m}(a)$ на единичной сфере $\sum\left|a_{s}\right|^{2}=1$ связаны соотношением $\Omega_{m}=\omega_{m}^{-1}=\lambda_{\max }\left(G_{m}\right)$.

Далее, с помощью четного продолжения с левой на правую полуось и с учетом определений величины $\psi_{m}(a)$ (см. $\left.(5),(6)\right)$ и матриц $P_{m}$ убеждаемся, что справедливо тождество

$$
\min \left\{\|y(x)\|_{W_{2}^{m}\left(\mathbb{R}_{-}^{1}\right)}^{2}: y^{(s-1)}(0)=a_{s}, s \in\{1, \ldots, m\}\right\}=\psi_{m}\left(-P_{m} a\right),
$$

из которого с учетом следствия 1 , в свою очередь, вытекает

СледствиЕ 3. Норма оператора наилучщего продолжения $T_{m}: W_{2}^{m}\left(\mathbb{R}_{-}^{1}\right) \rightarrow$ $W_{2}^{m}\left(\mathbb{R}^{1}\right)$ выражается формулой

$$
\tau_{m}=\sqrt{1+\frac{\Omega_{m}}{\omega_{m}}}=\sqrt{1+\lambda_{\max }^{2}\left(G_{m}\right)} .
$$

ЗАМЕчАНИЕ. Ясно, что величина $\Omega_{m}^{1 / 2}$ есть наименьшая возможная норма операторов экстраполяции $A_{m}^{(2)} \rightarrow W_{2}^{m}\left(\mathbb{R}_{+}^{1}\right)$, где $A_{m}^{(2)}-$ пространство векторов начальных значений $\left(f(0), f^{\prime}(0), \ldots, f^{(m-1)}(0)\right)$ с евклидовой нормой. В. Н. Габушиным [5] (см. также [6, 2.4.5]) получены явные (однако не эффективные) формулы для наибольших возможных значений в нуле промежуточных производных, т.е. чисел

$$
\Gamma_{s, m}^{+}:=\max \left\{\left|y^{(s)}(0)\right|:\|y\|_{W_{2}^{m}\left(\mathbb{R}_{+}^{1}\right)}=1\right\}, \quad s \in\{0,1, \ldots, m-1\} .
$$

Эти константы, как можно показать с учетом леммы 3 , выражаются через диагональные элементы матрицы $G_{m}$, а именно $\Gamma_{s, m}^{+}=\left(g_{s+1, s+1}^{(m)}\right)^{1 / 2}$. Л. В. Тайков доказал (см. [7] и $[6,2.4 .4])$, что числа, отличающиеся от $(20)$ взятием нормы не в $W_{2}^{m}\left(\mathbb{R}_{+}^{1}\right)$, а в $W_{2}^{m}\left(\mathbb{R}^{1}\right)$, выражаются простыми конструктивными формулами: $\Gamma_{s, m}=(2 m \sin ((2 s+1) \pi /(2 m)))^{-1 / 2}$.

\section{§4. Верхние оценки для матриц $\Phi_{m}$}

Лемма 4. Имеют место равенства $\ln \max \left|\varphi_{j, k}^{(m)}\right|=\left(K_{0}+\varepsilon_{m}\right) m$, где $K_{0}-$ константа, определенная в (4), а $\varepsilon_{m} \rightarrow 0$ при $m \rightarrow \infty$. 
ДокАЗАТЕЛЬСтво. Матричное равенство $\Phi V=E$ (индекс $m$ опускается) покоординатно записывается так (см. (11)):

$$
\sum_{s=1}^{m} \varphi_{j, s} \mu_{k}^{s-1}=\delta_{j, k}, \quad \text { где } \delta_{j, k}:=0(j \neq k), \delta_{k, k}:=1,
$$

а отсюда следует, что элементы каждой строки $\left\{\varphi_{j, s}\right\}$ матрицы $\Phi$ суть коэффициенты алгебраических интерполяционных многочленов Лагранжа степени $m-1$

$$
L_{m-1, j}(z):=\sum_{s=1}^{m} \varphi_{j, s} z^{s-1}, \quad \text { таких, что } L_{m-1, j}\left(\mu_{k}\right)=0, k \neq j, L_{m-1, j}\left(\mu_{j}\right)=1 \text {, }
$$

и, значит, эти многочлены могут быть записаны в виде

$$
L_{m-1, j}(z)=\prod_{k \neq j} \frac{z-\mu_{k}}{\mu_{j}-\mu_{k}}=\left(\prod_{k \neq j} \frac{1}{\mu_{j}-\mu_{k}}\right) \prod_{k \neq j}\left(z-\mu_{k}\right) .
$$

Модуль первого сомножителя, зависящего только от $m, j$ и не зависящего от $z$, как явствует из геометрических соображений, при фиксированном $m$ достигает наибольшего значения, когда $\mu_{j}$ максимально приближается к точке -1 . Следовательно, при нечетном $m, m=2 r+1$, поскольку $\mu_{r+1}=-1$, для любого $j \in\{1, \ldots, m\}$ справедлива оценка

$$
A_{m, j}:=\prod_{k \neq j}\left|\frac{1}{\mu_{j}-\mu_{k}}\right| \leqslant A_{2 r+1, r+1}=\prod_{s=1}^{r} \frac{1}{\left|\mu_{s}+1\right|^{2}}=\prod_{s=1}^{r}\left(2 \sin \frac{\pi s}{2 m}\right)^{-2} .
$$

Аналогично, для четного $m=2 r$ имеем при всех $j \in\{1, \ldots, m\}$

$$
A_{m, j} \leqslant A_{2 r, r}=A_{2 r, r+1}=\frac{1}{\sqrt{2}} \prod_{s=1}^{r-1}\left(2 \sin \frac{\pi s}{2 m}\right)^{-2} .
$$

Таким образом, при всех $m$ получено соотношение

$$
A_{m}:=\max _{j} A_{m, j}=A_{m,[m / 2]+1} \leqslant \prod_{s=1}^{[m / 2]}\left(2 \sin \frac{\pi s}{2 m}\right)^{-2} .
$$

Для случая $m=2 r+1, k=r+1$, группируя во втором сомножителе в $(23)$ пары скобок, соответствующие комплексно-сопряженным корням, получаем

$$
\prod_{k \neq r+1}\left(z-\mu_{k}\right)=\prod_{s=1}^{r}\left(z^{2}-\left(2 \operatorname{Re} \mu_{s}\right) z+1\right)=: \widetilde{L}_{2 r, r+1}(z)=\sum_{s=0}^{2 r} \tilde{\varphi}_{r, s} z^{s} .
$$

Все коэффициенты данного многочлена положительны; значит, их сумма равна значению многочлена при $z=1$, т.е.

$$
B_{2 r+1, r+1}:=\sum_{s=0}^{2 r}\left|\tilde{\varphi}_{r, s}\right|=\sum_{s=0}^{2 r} \tilde{\varphi}_{r, s}=\widetilde{L}_{2 r, r+1}(1)=\prod_{k \neq r+1}\left(1-\mu_{k}\right)=\prod_{s=1}^{r}\left(2 \cos \frac{\pi s}{2 m}\right)^{2} .
$$

Здесь мы снова использовали геометрические соображения, связанные с расположением корней, и приняли во внимание тот факт, что при $\mu$, лежащем на единичной окружности, векторы $1-\mu$ и $1+\mu$ ортогональны.

2 Функциональный анализ и его приложения, т. 36, вып. 2 
Далее, если $m$ нечетно, $m=2 r+1$, но $j<r+1$, то

$$
\widetilde{L}_{2 r, j}(z)=(z+1) \prod_{s=1}^{r} j^{j^{\prime}}\left(z^{2}-2\left(\operatorname{Re} \mu_{s}\right) z+1\right),
$$

где верхний индекс $j^{\prime}$ означает, что сомножитель с $s=j$ отброшен, и, следовательно,

$$
B_{2 r+1, j}=B_{2 r+1,2 r+1-j} \leqslant 2 \prod_{s=1}^{r} j^{j^{\prime}}\left(2 \cos \frac{\pi s}{2 m}\right)^{2} \leqslant B_{2 r+1, r+1},
$$

так как $(2 \cos (\pi j /(2 m)))^{2}>2$, поскольку $\pi j /(2 m)<\pi / 4$.

Для четного $m, m=2 r$, и $j \leqslant r$ аналогично получаем

$$
B_{2 r, j}=B_{2 r, 2 r-1-j} \leqslant 2 \prod_{s=1}^{r} j^{j^{\prime}}\left(2 \cos \frac{\pi(2 s-1)}{4 m}\right)^{2} \leqslant 4 \prod_{s=1}^{r}\left(2 \cos \frac{\pi s}{2 m}\right)^{2} .
$$

Соединяя (31), (30) и (26), получаем при всех $m$ неравенство

$$
B_{m}:=\max _{j} B_{m, j} \leqslant 4 \prod_{s=1}^{[m / 2]}\left(2 \cos \frac{\pi s}{2 m}\right)^{2},
$$

что вместе с (23) и (26) дает оценку для суммы модулей элементов любой строки матрицы $\Phi_{m}$ :

$$
\sum_{k=1}^{m}\left|\varphi_{j, k}^{(m)}\right| \leqslant A_{m} B_{m} \leqslant 4 \prod_{s=1}^{[m / 2]}\left(\operatorname{ctg} \frac{\pi s}{2 m}\right)^{2}
$$

Переходя в (33) к логарифмам, получаем для всех $m, j$ неравенство

$$
\ln \max _{j, k}\left|\varphi_{j, k}^{(m)}\right| \leqslant \ln 4+2 \sum_{s=1}^{[m / 2]} \ln \left(\operatorname{ctg} \frac{\pi s}{2 m}\right) .
$$

Далее, учитывая монотонное убывание функции $\ln (\operatorname{ctg} x)$ на интервале $(0, \pi / 4)$, запишем неравенства между интегральной суммой Римана и интегралами

$$
\int_{\pi /(2 m)}^{\pi / 4} \ln (\operatorname{ctg} x) d x \leqslant \frac{\pi}{2 m} \sum_{s=1}^{[m / 2]} \ln \left(\operatorname{ctg} \frac{\pi s}{2 m}\right) \leqslant \int_{0}^{\pi / 4} \ln (\operatorname{ctg} x) d x,
$$

и, поскольку интеграл в левой части стремится к интегралу справа, мы получаем соотношение

$$
\sum_{s=1}^{[m / 2]} \ln \left(\operatorname{ctg} \frac{\pi s}{2 m}\right)=\left(\frac{2 m}{\pi}+o(m)\right) \int_{0}^{\pi / 4} \ln (\operatorname{ctg} x) d x .
$$

Наконец, из (25) и (30) следует нижняя оценка

$$
\ln \max _{j, k}\left|\varphi_{j, k}^{(m)}\right| \geqslant \ln \left|\sum_{k=1}^{m} \varphi_{[m / 2]+1, k}^{(m)}\right|-\ln m \geqslant 2 \sum_{s=1}^{[m / 2]} \ln \operatorname{ctg} \frac{\pi s}{2 m}+o(m),
$$

из которой в соединении с $(32)$, (36) и определением (4) числа $K_{0}$ вытекает утверждение леммы 4. 


\section{$\S 5$. Оценки наименьшего собственного значения матрицы $Q_{m}$}

Следующее утверждение, относящееся к матрицам, определенным формулой (9), представляет также самостоятельный интерес.

ЛЕМма 5. $\ln \lambda_{\min }\left(Q_{m}\right) \approx-K_{0} m, m \rightarrow \infty$.

ДокАЗАТЕЛЬСтво. Пусть $m_{o}$ и $m_{e}$-число нечетных и соответственно четных натуральных чисел, не превосходящих $m$, т. е. $m_{o}:=[(m+1) / 2], m_{e}:=[m / 2]$, так что $m_{o}+m_{e}=m$. Учитывая, что $q_{j, k}^{(m)}=0$, если $j$ и $k-$ числа разной четности, мы заключаем, что в базисе $\left(e_{1}, e_{3}, \ldots, e_{2 m_{o}-1} ; e_{2}, e_{4}, \ldots, e_{2 m_{e}}\right)$ получаемом перестановкой стандартного базиса $\left(e_{1}, e_{2}, e_{3}, \ldots, e_{m}\right)$, матрица $Q_{m}$ становится блочно-диагональной,

$$
Q_{m}=\left(\begin{array}{cc}
Q^{(o)} & 0 \\
0 & Q^{(e)}
\end{array}\right), \quad q_{j, k}^{(o)}:=\frac{-2}{\mu_{2 j-1}+\bar{\mu}_{2 k-1}}, \quad q_{j, k}^{(e)}:=\frac{-2}{\mu_{2 j}+\bar{\mu}_{2 k}},
$$

где диагональные блоки $Q^{(o)}$ и $Q^{(e)}$ имеют размеры $m_{o}$ и $m_{e}$ соответственно.

Утверждение леммы 5 равносильно следующим соотношениям для наибольших собственных значений матриц $U_{m_{o}}^{(o)}, U_{m_{e}}^{(e)}$ (порядка $m_{o}, m_{e}$ ), обратных к $Q^{(o)}$ и $Q^{(e)}$ соответственно:

$$
\ln \lambda_{\max }\left(U_{m_{o}}^{(o)}\right) \approx K_{0} m, \quad \ln \lambda_{\max }\left(U_{m_{e}}^{(e)}\right) \approx K_{0} m, \quad m \rightarrow \infty .
$$

Из формул для определителей Коши $[8, \S 14]$ (см. также [9, п. 2], где следует положить $x_{r}=\bar{y}_{r}=\mu_{r}$ ) получаем явные выражения для диагональных элементов матриц $U_{m_{o}}^{(o)}, U_{m_{e}}^{(e)}$ :

$$
\begin{aligned}
& u_{k, k}^{(o)}=\frac{\left|\mu_{2 k-1}+\bar{\mu}_{2 k-1}\right|}{2} \prod_{j \neq k}\left|\frac{\mu_{2 j-1}+\bar{\mu}_{2 k-1}}{\mu_{2 j-1}-\bar{\mu}_{2 k-1}}\right|^{2}, \\
& u_{k, k}^{(e)}=\frac{\left|\mu_{2 k}+\bar{\mu}_{2 k}\right|}{2} \prod_{j \neq k}\left|\frac{\mu_{2 j}+\bar{\mu}_{2 k}}{\mu_{2 j}-\bar{\mu}_{2 k}}\right|^{2} .
\end{aligned}
$$

Произведения по $j$ в (40) оцениваются точно так же, как произведения в доказательстве леммы 4 (см. вывод оценок (26), (32), (33)). Вводя обозначения $k_{o}:=\left[m_{o} / 2\right]+1, k_{e}:=\left[m_{e} / 2\right]+1$, для произведений по нечетным индексам получим

$$
w_{k}:=\prod_{j \neq k}\left|\frac{\mu_{2 j-1}+\bar{\mu}_{2 k-1}}{\mu_{2 j-1}-\bar{\mu}_{2 k-1}}\right| \leqslant \prod_{j \neq k}\left|\frac{\mu_{2 j-1}-1}{\mu_{2 j-1}+1}\right| \leqslant \prod_{s=1}^{k_{o}}\left(2 \operatorname{ctg} \frac{\pi s}{m}\right)^{2}=: p^{(o)},
$$

причем при выборе $k=k_{o}$ будет выполняться неравенство $w_{k} \geqslant p^{(o)} / 4$.

Аналогично для произведений по четным индексам имеем

$$
\prod_{j \neq k}\left|\frac{\mu_{2 j}+\bar{\mu}_{2 k}}{\mu_{2 j}-\bar{\mu}_{2 k}}\right| \leqslant \prod_{s=1}^{k_{e}}\left(2 \operatorname{ctg} \frac{\pi s}{m}\right)^{2} \leqslant 4 \prod_{j \neq k_{e}}\left|\frac{\mu_{2 j}+\bar{\mu}_{2 k_{e}}}{\mu_{2 j}-\bar{\mu}_{2 k_{e}}}\right| .
$$


Учитывая еще, что $\max \left|\mu_{k}+\bar{\mu}_{k}\right| \leqslant 2$, тогда как оба числа $\left|\mu_{2 k_{o}-1}+\bar{\mu}_{2 k_{o}-1}\right|$ и $\left|\mu_{2 k_{e}}+\bar{\mu}_{2 k_{e}}\right|$ близки к 2 при всех достаточно больших $m$, мы заключаем, что максимум диагональных элементов матриц $U_{m_{o}}^{(o)}, U_{m_{e}}^{(e)}$ равен по порядку

$$
\max _{k} u_{k, k}^{(o)} \asymp \max _{k} u_{k, k}^{(e)} \asymp u_{[m / 4],[m / 4]}^{(o)} \asymp u_{[m / 4],[m / 4]}^{(e)} \asymp \prod_{s=1}^{[m / 4]}\left(\operatorname{ctg} \frac{\pi s}{m}\right)^{4} .
$$

Из рассуждений предыдущего параграфа (см. цепочку (35)-(37)) следует, что

$$
\ln \max _{k} u_{k, k}^{(o)} \approx \max _{k} u_{k, k}^{(e)} \approx 4 \sum_{s=1}^{[m / 4]} \ln \left(\operatorname{ctg} \frac{\pi s}{m}\right) \approx \frac{4}{\pi} \mathbf{G} m=K_{0} m, \quad m \rightarrow \infty,
$$

а поскольку для любой положительно определенной матрицы $U_{n}$ порядка $n$ выполняются неравенства $\ln \max u_{k, k} \leqslant \ln \lambda_{\max }\left(U_{n}\right) \leqslant \ln \sum u_{k, k} \leqslant \ln n+\ln \max u_{k, k}$, из (44) вытекает (39).

Доказательство леммы 5 закончено.

ЗАмЕЧАНИЕ. Отметим, что, согласно (9), имеет место тождество $Q_{m}=\widetilde{Q}_{m}+$ $\widehat{Q}_{m}$, где $\widetilde{Q}_{m}-$ матрица с элементами $\tilde{q}_{j, k}:=-2\left(\mu_{j}+\bar{\mu}_{k}\right)^{-1}$, а $\widehat{Q}_{m}:=P_{m} \widetilde{Q}_{m} P_{m}$. Из рассуждений, примененных в доказательстве леммы 5 , следует, что

$$
\ln \lambda_{\min }\left(\widetilde{Q}_{m}\right)=\ln \lambda_{\min }\left(\widehat{Q}_{m}\right) \approx-2 K_{0} m,
$$

тогда как логарифм наименьшего собственного значения их суммы, согласно лемме 5 , убывает в два раза медленнее: $\ln \lambda_{\min }\left(Q_{m}\right) \approx-K_{0} m$.

\section{§6. Завершение доказательства теоремы}

Из определения матрицы $G_{m}$ (см. формулу $\left.(15)\right)$ вытекает верхняя оценка

$$
\left(G_{m} a, a\right)=\left(i^{m-1} V_{m} P_{m} \Phi_{m} a, P_{m} S_{m} a\right) \leqslant m^{2}\left(\max _{j, k}\left|\varphi_{j, k}^{(m)}\right|\right)|a|^{2},
$$

откуда с учетом определения (19), леммы 4 (см. (36)) и формулы (4) следует неравенство

$$
\ln \tau_{m} \leqslant \ln \lambda_{\max }\left(G_{m}\right)+o(1) \leqslant \ln \max _{j, k} \ln \left|\varphi_{j, k}^{(m)}\right|+2 \ln m+O(1) \leqslant K_{0} m+o(m),
$$

и нам теперь остается лишь убедиться в том, что справедливо и обратное к (46) неравенство.

Из формулы (12), определяющей матрицу $G_{m}$, следует цепочка неравенств

$$
\left(G_{m} a, a\right)=\left(\Phi_{m}^{*} Q_{m} \Phi_{m} a, a\right)=\left(Q_{m} \Phi_{m} a, \Phi_{m} a\right) \geqslant \lambda_{\min }\left(Q_{m}\right)\left(\Phi_{m} a, \Phi_{m} a\right) .
$$

Выберем здесь в качестве $а$ единичный вектор $\tilde{a},\|\tilde{a}\|=1$, с равными координатами $\tilde{a}_{j}=1 / \sqrt{m}$, для которого в силу леммы 4 (см. (37)) выполняется неравенство

$$
\left\|\Phi_{m} \tilde{a}\right\| \geqslant m^{-1 / 2}\left|\sum_{k=1}^{m} \varphi_{[m / 2]+1, k}\right| \geqslant e^{\left(K_{0}-\varepsilon_{m}\right) m} .
$$

Тогда из последних двух соотношений и леммы 5 получаем

$$
\ln \lambda_{\max }\left(G_{m}\right) \geqslant \ln \left(G_{m} \tilde{a}, \tilde{a}\right) \geqslant \ln \lambda_{\min }\left(Q_{m}\right)+2 \ln \left\|\Phi_{m} \tilde{a}\right\| \geqslant\left(K_{0}-\tilde{\varepsilon}_{m}\right) m+o(m)
$$


В заключение отметим, что векторы $\tilde{a}$ начальных данных с равнылми координатами обеспечивают рост логарифма нормы экстраполяции в $W_{2}^{m}\left(\mathbb{R}_{+}^{1}\right)$, асимптотически равный $K_{0} m / 2$. Однако (при нечетном $m$ ) экстраполяция этих начальных условий на отрицательную полуось имеет норму в $W_{2}^{m}\left(\mathbb{R}_{-}^{1}\right)$, в точности равную 1. Поэтому остается неясным, какие наборы начальных условий дают разность логарифмов норм экстраполяций в $W_{2}^{m}\left(\mathbb{R}_{+}^{1}\right)$ и $W_{2}^{m}\left(\mathbb{R}_{-}^{1}\right)$, эквивалентную $K_{0} m$.

\section{ЛиТЕРАТУРА}

1. Буренков В. И., Горбунов А. Л. Точные оценки наименьших норм операторов продолжения для соболевских пространств. Изв. РАН, сер. матем., 61, № 1, 3-44 (1997).

2. Burenkov V. I., Kalyabin G. A. Lower estimates of the norm of extension operators for Sobolev spaces on the halfline. Math. Nachr., 218, 19-23 (2000).

3. Двайт Г. Б. Таблицы интегралов и другие математические формулы. Наука, М., 1978.

4. Klinger $A$. The Vandermonde matrix. Amer. Math. Monthly, 74, 571-574 (1967).

5. Габушин B. H. Точные константы в неравенствах между производными функции. Матем. заметки, 4, вып. 2, 221-232 (1968).

6. Тихомиров В. М. Некоторые вопросы теории приближений. Изд-во МГУ, М., 1976.

7. Тайков Л. В. Неравенства колмогоровского типа и наилучшие формулы численного дифференцирования. Матем. заметки, 4, № 2, 233-238 (1968).

8. Ахиезер Н. И. Лекции по теории аппроксимации. Наука, М., 1965.

9. Калябин $Г$. А. Асимптотика наименьших собственных значений матриц типа Гильберта. Функц. анализ и его прил., 35, вып. 1, 80-84 (2001).

Самарская гуманитарная академия,

Самарский государственный аэрокосмический университет e-mail: klgnaa@mail.ru, kalyabin@mb.ssau.ru, kalyabin@lycos.com
Поступило в редакцию 19 октября 2001 г. 